the top layer format using a prototype online authoring tool tailored for the format. Participants will at the end of the session be asked to provide feedback on the novel format, specifically on relevance, comprehension, likability and feasibility of production. Feedback is collected using a multiple-choice survey with clickers in addition to a final discussion

\section{WS IMPROVING GUIDELINE IMPLEMENTABILITY WITH GUIDE-M (GUIDELINE IMPLEMENTABILITY FOR DECISION EXCELLENCE MODEL): AN INTERACTIVE WORKSHOP}

${ }^{1} M$ Kastner, ${ }^{2} \mathrm{~J}$ Makarski, ${ }^{1} \mathrm{~L}$ Hayden, ${ }^{2} \mathrm{~L}$ Durocher, ${ }^{1} \mathrm{~A}$ Chatterjee, ${ }^{1} \mathrm{O}$ Bhattacharyya, ${ }^{2} \mathrm{M}$ Brouwers. 'Li Ka Shing Knowledge Institute of St. Michael's Hospital, Toronto, Canada; ${ }^{2}$ Department of Oncology, McMaster University, Hamilton, Canada

\section{0:1136/bmjgs-2013-002293.27}

Background We developed a framework of guideline uptake called GuIDE-M (Guideline Implementability for Decision ExcellenceModel) based on an extensive literature review. It describes four domains covering guideline content to optimise the implementability of recommendations (Stakeholder development, Evidence synthesis, Considered Judgement and Feasibility) and two domains related to communication of content (Language and Format).

Objectives/Goal (1) To learn about GuIDE-M, (2) To conduct an assessment of participants' current use of the GuIDE-M domains in guideline development or assessment and (3) To determine priorities for tool development to operationalize GuIDE-M domains.

Target Group, Suggested Audience Guideline developers, guideline users and researchers. [5]

Description of the Workshop and Methods used to Facilitate Interactions (1) Introduction (15 minutes). A brief foundational overview of GuIDE-M. (2) Facilitated Assessment (60 minutes). Participants will break into small groups to discuss one or more of the domains in GuIDE-M. There they will (a) conduct a more detailed review of the domain, (b) assess the extent to which their guideline-related activities align with GuIDE-M principles, (c) reflect on the extent to which improving in the area is a priority, (d) discuss methods and available tools to operationalize the domain concepts, and (e) explore the types of tool(s) that should be developed to incorporate domain concepts into guideline development. Participants will be invited to remain involved as evaluators, pilot-testers and developers of these tools. The facilitated assessment will happen twice $(2 \times 30$ minutes $)$ to allow participants to focus on two of the GuIDE-M domains. (3) Wrap-Up (15 minutes).

\section{WS SOFTWARE TOOLS AND ACTION STATEMENT PROFILES TO FACILITATE GUIDELINE DEVELOPMENT}

${ }^{1} \mathrm{R}$ Shiffman, ${ }^{2} \mathrm{R}$ Rosenfeld. 'Yale University School of Medicine, New Haven, CT, USA; ${ }^{2}$ American Academy of Otolaryngology - Head and Neck Surgery, Alexandria, VA, USA

\section{0:1136/bmjgs-2013-002293.28}

Background Despite the importance of guideline recommendations being "actionable", many current guidelines fail to meet standards for clarity, transparency, and implementability. These deficiencies contribute to failure of guidelines to influence care. Objectives/Goals Attendees will • Learn how BRIDGE-Wiz software can lead developers to devise clear and actionable statements linked to appropriate indicators of evidence quality and recommendation strength; - Learn how eGLIA software can help to identify obstacles to successful implementation. - Learn how key action statements and action statement profiles can be used to support transparency of the guideline development process and promote successful implementation.

Target Group, Suggested Audience Guideline developers, guideline implementers

Description of the Workshop and of the Methods used to Facilitate Interactions Following a brief overview of IOM standards for trustworthy guidelines and common problems in creating actionable recommendations, the facilitators will lead the "panel" through dynamic creation of a guideline recommendation using BRIDGE-Wiz. Attendees will function as a guideline development group responding to prompts from the software and interacting with the facilitators and one another. Facilitators will next provide a brief demonstration of eGLIA software appraising selected recommendations to identify implementability challenges. With audience participation, recommendations will be appraised and conflicting appraisals reconciled to create an implementability report. Finally, the construction and use of action statement profiles will be discussed. Action statement profiles summarise information about each recommendation and make explicit and transparent the process by which evidence and opinion are transformed into recommendations about appropriate care.

\section{WS EVIDENCE TO RECOMMENDATIONS FRAMEWORKS: DIAGNOSIS}

${ }^{1} \mathrm{H}$ Schünemann, ${ }^{2} \mathrm{P}$ Alonso, ${ }^{3 P} \mathrm{P}$ Bossuyt, ${ }^{1} \mathrm{~J}$ Brozek, ${ }^{4} \mathrm{M}$ Leeflang, ${ }^{4} \mathrm{G}$ Deurenberg-Gopalakrishna, ${ }^{4} \mathrm{M}$ Langendam, ${ }^{5} \mathrm{M}$ Koster, ${ }^{1} \mathrm{R}$ Mustafa, ${ }^{1} \mathrm{~N}$ Santesso. ${ }^{1} \mathrm{M}$ CMaster University, Hamilton, Canada; ${ }^{2}$ Institute of Biomedical Research (IIIB Sant Pau), Barcelona, Spain; ${ }^{3}$ University of Amsterdam, Amsterdam, Netherlands; ${ }^{4}$ Academic Medical Center, Amsterdam, Netherlands, ${ }^{5}$ Kaiser Permanente, California, United States

\section{0:1136/bmjgs-2013-002293.29}

Background Moving from evidence to recommendations (EtR) in guideline development requires balancing evidence quality with the benefits and harms of interventions, patient preferences, and resource and cost considerations. Developing recommendation about diagnostic tests and strategies is particularly challenging and requires tackling complex challenges, different than those needed for therapeutic interventions. The GRADE Working Group, has developed an approach to integrate these factors into development of clinical practice recommendations that is currently further defined in the DECIDE (Developing and Evaluating Communication Strategies to Support Informed Decisions and Practice Based on Evidence) project. This workshop will introduce this approach and evaluate the EtR framework based on examples and hands-on exercises.

Objectives To learn how to use and evaluate the EtR framework for diagnostic questions.

Target Group, Suggested Audience Guideline developers, systematic reviewers, clinicians.

Description of the Workshop and of the Methods used to Facilitate Interactions This workshop provides a brief didactic overview of GRADE for diagnostic questions. Each group will use a systematic review and a partially pre-filled EtR framework. During the small group work, participants will discuss challenges and advantages of the approach. Participants will then apply these concepts in small groups to develop a recommendation based on the workshop material; there will be a plenary to provide feedback that will help to enhance the work and provide opportunities for collaboration. 